\title{
CORRESPONDENCE
}

\section{To THE EDITOR}

\section{Mechanisms of Action of IVIG in Adult Onset Rasmussen's Encephalitis}

\section{Re: Can J. Neurol. Sci. 2006;33:302-305 Rasmussen's Encephalitis in a 58-Year-Old Female: Still a Variant?}

In your August issue Hunter et $\mathrm{al}^{1}$ described the case of the oldest patient with confirmed diagnosis of Rasmussen's encephalitis to date. In the article they suggest a disease mechanism that is similar to myasthenia gravis. Whereas in myasthenia gravis the auto-reactive antibodies are against the acetylcholine receptor, ${ }^{2}$ it is becoming clear that in certain cases of Rasmussen's encephalitis, the presence of auto-reactive antibodies against the glutamate receptor results in neurological deficits. Importantly, the authors show and recommend that intravenous immunoglobulin (IVIG) therapy may be an effective first line treatment of adult onset Rasmussen's encephalitis. ${ }^{1}$

While no brand of IVIG currently carries FDA indications for applications in the central nervous system, there is widespread use of IVIG for many neurological disorders with a range of purported mechanisms. ${ }^{3}$ With regard to myasthenia gravis, IVIG treatment is believed to be effective, ${ }^{2}$ and there are three potential mechanisms of action that could be applied to explain the effect of IVIG therapy in this disease state. ${ }^{3}$ Because of the potential similarities in immune dysregulation between myasthenia gravis and Rasmussen's encephalitis, it is possible to extrapolate mechanisms of action for IVIG in treating patients with certain variants of Rasmussen's encephalitis. First, IVIG may contain anti-idiotype antibodies that are able to sequester the auto-reactive antibodies that cause Rasmussen's encephalitis (Figure 1A). Second, IVIG may effectively dilute the concentration of auto-reactive antibodies to a level that prevents both variable region mediated binding to the antigen and constant region binding to the $\mathrm{Fc}$ receptor (Figure 1B). This second mechanism may also incorporate a reduction in endogenous antibody production. ${ }^{2}$ Third, infusion with IVIG may saturate the Fc receptor and competitively inhibit autoreactive antibody-mediated immune function in a dominantnegative manner (Figure 1C).

It may be that one of these mechanisms of actions is independently responsible for the therapeutic effect of IVIG in Rasmussen's encephalitis; or it may be that there is a synergistic effect between the mechanisms. Regardless, if these mechanisms of action prove true for IVIG therapy in myasthenia gravis, and a variant of Rasmussen's encephalitis is shown to be dependent upon the existence of auto-reactive anti-glutamate receptor antibodies, then the mechanism of action of IVIG therapy for both myasthenia gravis and particular variants of Rasmussen's encephalitis will likely be similar.
Unfortunately, it is not possible to leverage the case reports in the current literature to extrapolate which of the three proposed mechanisms is most appropriate. Nonetheless, because of the reports of auto-reactive antibodies against the glutamate receptor, and the similarities with myasthenia gravis, there is likely some element of anti-idiotype action occurring during IVIG treatment of Rasmussen's encephalitis. ${ }^{4}$ To test this hypothesis it would be valuable to determine if there are antiidiotype antibodies to the auto-reactive glutamate receptor antibody in IVIG preparations. If there are appropriate antiidiotype antibodies in IVIG, it may be possible to more effectively treat Rasmussen's encephalitis with a monoclonal anti-idiotype antibody. Moreover, it may be possible to use such an anti-idiotype antibody to intelligently design an aptamerbased therapy that exhibits high affinity and high specificity as well as lower cross-reactivity as compared to monoclonal antibody preparations. Such tailored treatments would allow a more effective treatment of this rare disorder.

Eric J. Buenz North Carolina, USA

Charles L. Howe Minnesota, USA

\section{REFERENCES}

1. Hunter GR, Donat J, Pryse-Phillips W, Harder S, Robinson CA. Rasmussen's encephalitis in a 58-year-old female: still a variant? Can J Neurol Sci. 2006; 33(3):302-5.

2. Howard JF Jr. Intravenous immunoglobulin for the treatment of acquired myasthenia gravis. Neurology. 1998; 51(6 Suppl 5): S30-6.

3. Dalakas MC. Mechanisms of action of IVIg and therapeutic considerations in the treatment of acute and chronic demyelinating neuropathies. Neurology. 2002; 59(12 Suppl 6): S13-21.

4. Krolick KA, Zoda TE, Thompson PA. Examination of characteristics that may distinguish disease-causing from benign AChR-reactive antibodies in experimental autoimmune myasthenia gravis. Adv Neuroimmunol. 1994; 4(4):475-93. 


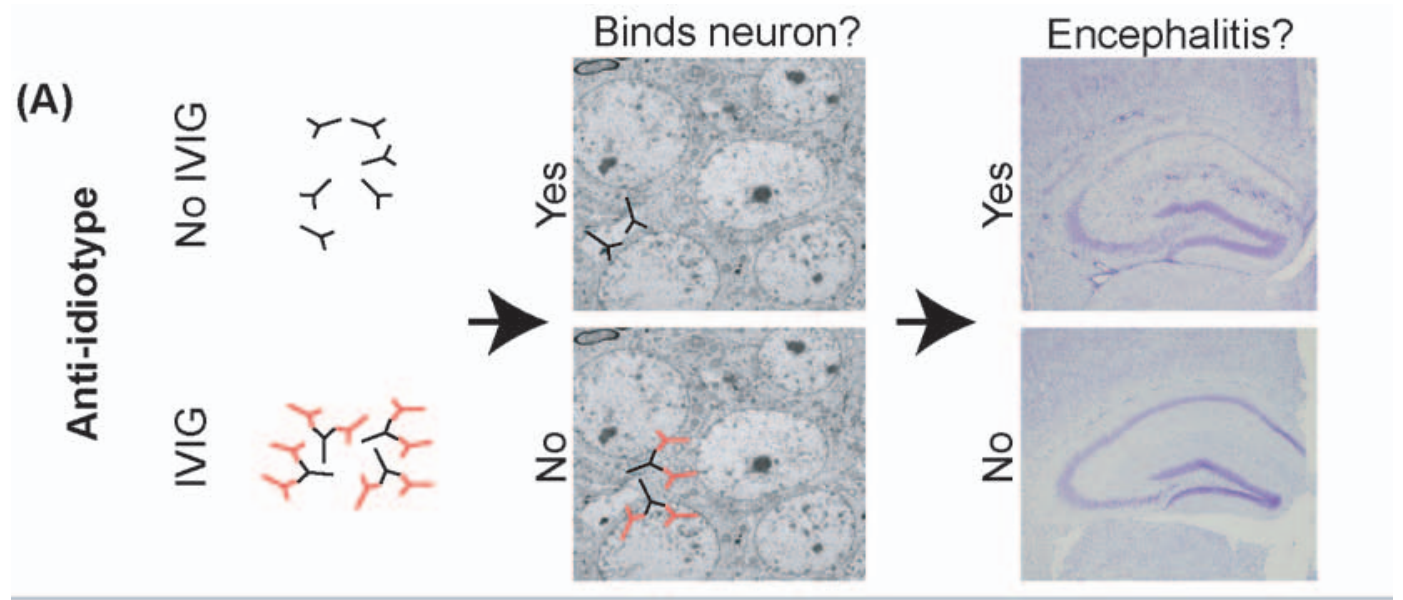

(B)
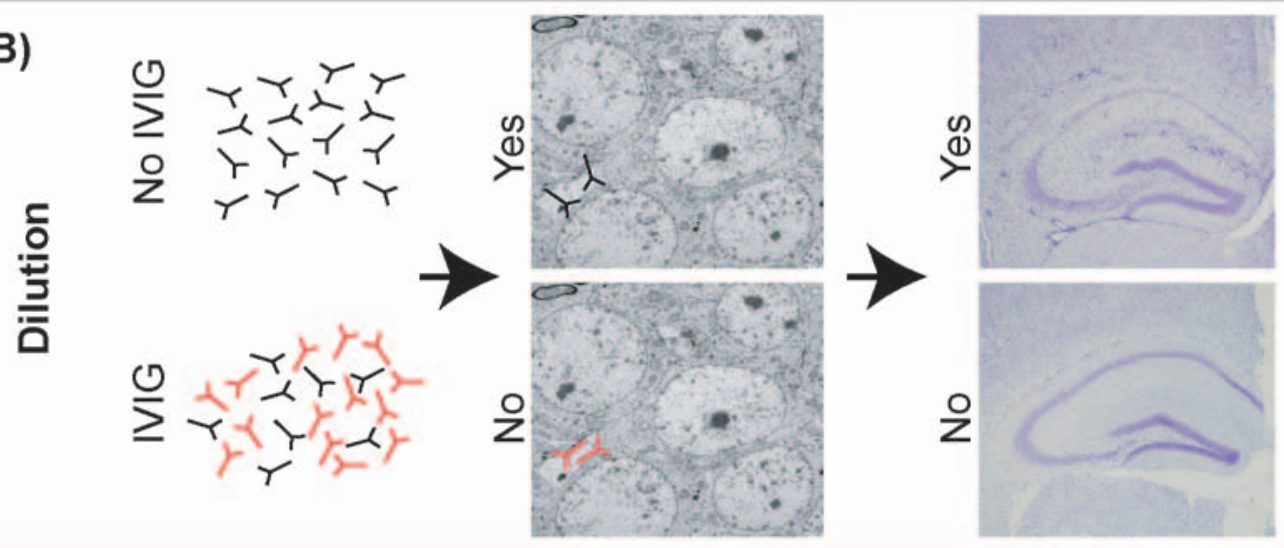

(C)
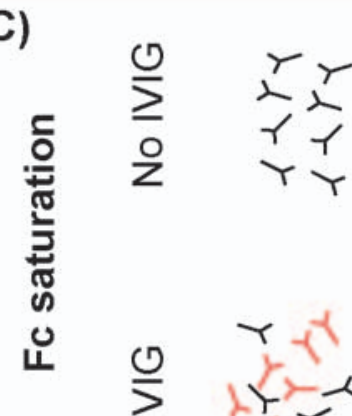

\section{Signals immune cell?}
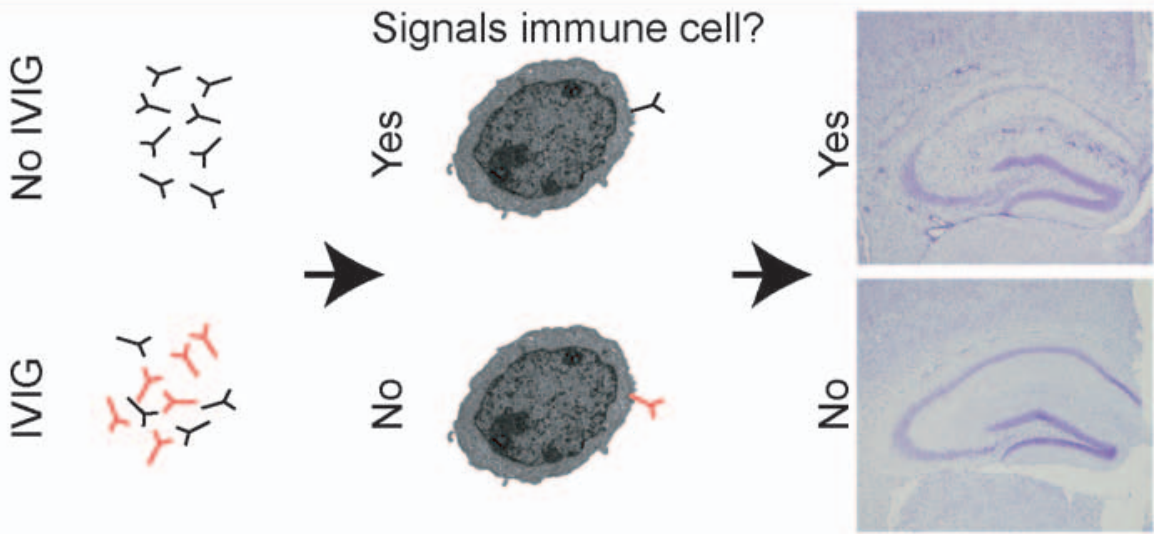

tEndogenous

Exogenous (IVIG)

Figure: Three models for the mechanism of action explaining the effect of IVIG seen in Rasmussen's encephalitis. A) The anti-idiotype mechanism sequesters the auto-reactive antibodies. The IVIG antibodies binding the variable region of the auto-reactive antibody do not allow the auto-reactive antibody to recognize self epitopes. $B)$ The dilution mechanism effectively reduces the concentration of auto-reactive antibodies. Because both the endogenous and IVIG-provided antibodies undergo degradation at the same rate, it is hypothesized that massive quantities of antibodies introduced through IVIG infusion will facilitate degradation of the auto-reactive antibodies and inhibit endogenous antibody production. Thus, while antibodies may be present in the CNS, these IVIG-introduced antibodies are not auto-reactive. C) The Fc saturation model is based on the premise of competitive inhibition of the $F_{c}$ receptor that is present on many immune cells. Because both the auto-reactive and the non-auto-reactive IVIG-supplied antibodies compete for the Fc receptor on immune cells, the infusion of IVIG limits the ability of the auto-reactive antibodies to activate the immune cells. 